\title{
STRONG WAVES FROM PULSARS AND MORPHOLOGIES IN SNRS
}

\author{
Gregory Benford \\ University of California, Irvine
}

Attilio Ferrari and Silvano Massaglia

Istituto di Fisica Generale dell'Universita'. Torino

\section{INTRODUCTION}

Canonical models for pulsars predict the emission of low--frequency waves of large amplitudes, produced by the rotation of a neutron star possessing a strong surface magnetic field. Pacini (1968) proposed this as the basic drain which yields to the pulsar slowing-down rate. The main relevance of the large amplitude wave (LAW) is the energetic link it provides between the pulsar and the surrounding medium. This role has been differently emphasized (Rees and Gunn, 1974; Ferrari, 1974), referring to absorption effects by relativistic particle acceleration and thermal heating, either close to the pulsar magnetosphere or in the nebula. It has been analyzed in the special case of the Crab Nebula, where observations are especially rich (Rees, 1971). As the Crab Nebula displays a cavity around the pulsar of dimension $\sim 10^{17} \mathrm{~cm}$, the function of the wave in sweeping dense gas away from the circumpulsar region is widely accepted. Absorption probably occurs at the inner edges of the nebula; i.e., where the wave pressure and the nebular pressure come into balance. Ferrari (1974) interpreted the wisps of the Crab Nebula as the region where plasma absorption occurs, damping the large amplitude wave and driving "parametric" plasma turbulence, thus trasferring energy to optical radiation powering the nebula. The mechanism has been extended to interpret the specific features of the "wisps" emission (Benford et al.,1978). Possibly the wave fills the nebula completely, permeating the space outside filaments with electromagnetic energy, continuously accelerating electrons for the extended radio and optical emission (Rees, 1971).

The details of the interaction of a low-frequency wave with magnetospheric and circumstellar plasmas has been discussed by Dobrowolny and Ferrari (1976) and by Benford et al. (1978). Benford et al. (1982) propose a general scenario for the interaction between the strong electromagnetic wave and the surrounding medium. The aim is to derive which morphologies should be expected for a range of the physical parameters corresponding to different stages of remnant's evolution or location in the Galaxy. In particular they attempt to define which observations would allow indirect detection of a spinning neutron star in a supernova remnant, where the geometry is not favourable to direct observation of pulses. Following this analysis, it turns out that two scenarios can be envisioned, depending on the density of the circumpulsar region: i) low-density magnetosphere case (LDM), which leads to morphologies typical of Crab-like supernova remnants, and ii) high density magnetosphere case (HDM), which yields to objects like 499 
SS433 and compact, high-brightness sources. We summarize here the main results of this model in connection with possible observational checks.

\section{CRAB-LIKE SNRS}

Two classes of SNRs are usually recognized: shell-type SNRs (possibly caused by Type I SNs) and Crab-like SNRs or "plerions" (possibly due to Type II events) (Weiler and Panagia, 1978, 1981). Plerions may be supported by the activity of young, fast pulsars, and should be short lived $\left(\sim 2 \times 10^{4} \mathrm{yr}\right)$ from heavy braking. In most observed plerions the central object has not been detected as a pulsar. Beaming away from us may account for this; instead, energy loss occurs mostly through large amplitude wave mechanisms within a parsec of the object. If so, the only method of detection may be through non-thermal $\mathrm{X}$-ray emission or coherent (but unpulsed) radio radiation.

To illustrate the range of parameters needed, Benford et al. (1982) consider a sample of plerions detected in the radio with luminosity $\mathrm{L}_{\mathrm{r}}$. The total power emitted by a spinning magnetic dipole is

$$
\mathrm{L}_{\mathrm{d}} \approx 5 \times 10^{31} \mathrm{a}_{6}^{6} \quad \mathrm{~B}_{12}^{2} \mathrm{P}^{-4} \mathrm{erg} / \mathrm{sec}
$$

By equating the observed $L_{r}$ to $L_{d}$ one obtains a crude upper limit on the period, $P$. A minimum distance at which parametric instabilities can occur in a circumstellar plasma is then found. This is roughly the size of a non-thermal $\mathrm{X}$-ray feature arising from synchro-Compton processes as the wave decays. The expected angular sizes fall within the angular resolution of forthcoming $X$-ray satellites. To estimate $X$-ray luminosities we scale from $L_{r}$ to $L_{X}$ using the same ratio as the Crab in $2-6 \mathrm{keV}$ range. Benford et al.(1978) found the synchro-Compton radiation from the $\mathrm{Crab}$ in this range to be given by $\mathrm{w}_{\mathrm{x}} \cong 10^{37} \mathrm{n}_{\mathrm{x}} / \mathrm{n}_{\mathrm{w}}$ where $\mathrm{n}_{\mathrm{x}} / \mathrm{n}_{\mathrm{w}}$ is the ratio of electrons at $\gamma \approx 10^{8}$ to the bulk density carried by the wave, $\mathrm{n}_{\mathrm{w}}$. Setting $\mathrm{W}_{\mathrm{x}} \cong \mathrm{L}_{\mathrm{x}}, \mathrm{n}_{\mathrm{x}} / \mathrm{n}_{\mathrm{w}} \sim 10^{-4} \div 10^{-3}$. Parametric decay of the wave produces particle bunching, leading to coherent, antenna-like emission (Leboeuf et al., 1982). Thus some coherence may occur far from the central object, though the plasma frequencies in this region may be so low that the emission is at frequencies below the radio. It seems likely that coherence is best observed close to the central object.

As an interesting case, we finally recall that Seward and Harnden (1982) found pulsed $\mathrm{X}$-ray emission coming from a point source MSH 15-52. The observed period is $0.150 \mathrm{sec}$. The source appears to be near a small region of non-thermal diffuse $\mathrm{X}$-ray emission, probably powered by the pulsar itself, as it is typical of Crab-like SNRs. The diffuse $X$-ray luminosity is $L_{X} \cong 2 \times 10^{35} \mathrm{erg} / \mathrm{sec}$. If we suppose this is a pulsar, rather than a binary neutron star source, we can apply the method above to get an upper limit on the period in terms of the $\mathrm{X}$-ray luminosity. This yields $\mathrm{P} \cong 0.126 \mathrm{a}_{6}^{3 / 2} \mathrm{~B}_{12}^{1 / 2} \mathrm{sec}$, quite close to the observed $\mathrm{X}$-ray period.

\section{HIGH-BRIGHTNESS TEMPERATURE REMNANTS}

If the plasma beyond the light cylinder is too dense $\left(\gtrsim 10^{-8} / \mathrm{P}^{2} \mathrm{~cm}^{-3}\right)$, the wave will be absorbed in a few light cylinder radii, as the wave pressure does not exceed the plasma pressure (Dobrowolny et al., 1976). Computer simulations of Lebouef et al. (1982) show that, regardless of waveform, the large amplitude wave rapidly decays into 
a mixture of ultrarelativistic particles and waves. What is more, localized density spikes appear at the extrema of the wave magnetic field. Bunched electrons can radiate coherently.

We may expect that many spinning neutron stars will be surrounded by dense plasmas. This will be the case particularly if the pulsar has high linear speed and thus confronts fresh interstellar plasma constantly, so that it cannot evacuate a cavity. Such smothered magnetospheres should display the effects of wave decay beyond their light cylinder radii, and if the parametric decay involves particle bunching, a steady coherent emission should appear. This radiation will generally not be pulsed, since the bunches are not being swept around by a rigid magnetosphere. We can crudely estimate the brightness temperature of a light electron wind oscillating in the wave troughs. For the typical pulsar parameters, even if the wave decays parametrically in a distance L, it will radiate in radio wavelengths with brightness temperature

$$
\mathrm{T}_{\mathrm{b}} \approx 10^{19} \mathrm{~L}_{10} \nu_{9}^{-3} \gamma_{4}^{2} \mathrm{n}_{\sigma}, \mathrm{B}_{12}^{2} \mathrm{P}_{-1}^{-6} \quad{ }^{\circ} \mathrm{K}
$$

where $\gamma$ is the wind Lorentz factor, $\mathrm{n}$ its plasma density, and $\mathrm{B}$, a, $\mathrm{P}$ are, respectively, the surface magnetic field, the radius and the period of the spinning object. Such bright compact, non-pulsing objects should be sought by scintillation techniques (see Cordes and Dickey 1979, for a possible discovery of one of this kind of objects). If a dispersive smearing of a pulsed component can be eliminated, such a source will provide a signature of the large amplitude wave.

\section{REFERENCES}

Benford,G., Bodo,G. and Ferrari,A., (1978) Astron. Astrophys. 70, 213.

Benford,G., Ferrari,A. and Massaglia,S., (1982) Ap. J., submitted.

Cordes,J.M. and Dickey,J.M., (1979) Nature 281, 24.

Dobrowolny,M. and Ferrari,A., (1976) Astron. Astrophys. 47 , 97.

Ferrari,A., (1974) "Supernovae and Supernova Remnants", D.Reidel, 375.

Leboeuf,J.N., Ashorn-Abdalla,M., Tajima,T., Dawson,J.M., Coroniti,F.V. and Kennell,C.F., (1982), to be published.

Pacini,F., (1968) Nature 219, 145.

Rees,M.J., (1971) Nature 230 , 55.

Rees,M.J. and Gunn,J.E., (1974) Mon. Not. R. astr. Soc. 167, 1.

Seward,F.D. and Harnden,F.R., (1982) Ap. J., in press.

Weiler,K.W. and Panagia,N., (1978) Astron. Astrophys. $\underline{70}, 419$.

Weiler,K.W. and Panagia,N., (1980) Astron. Astrophys. 무, 269. 